\title{
Reconstrucción de roturas \\ inveteradas del tendón \\ distal del biceps brachii \\ con aloinjerto liofilizado \\ de tendón de Aquiles
}

\author{
P. J. Delgado ${ }^{(1)}$, J. I. García-López ${ }^{(1)}$, J. M. Abad ${ }^{(2)}$, F. García de Lucas ${ }^{(3)}$ \\ Hospital FREMAP. MAJadahonda, Madrid, España. UNIDAD DE Miembro Superior \\ (1) MÉdico ADJUNTO \\ (2) JefE de Servicio de Cirugía Ortopédica y TRaumatología \\ (3) DiRECTOR MÉdICO NACIONAL
}

Correspondencia:

Dr. Pedro J. Delgado Serrano

Unidad de Cirugía de la Mano y Miembro Superior

Hospital Universitario Madrid Montepríncipe

Avda. Montepríncipe, 25

28660 Boadilla del Monte, Madrid, España

E-mail: pedrojdelgado@me.com

Objetivo: Las roturas inveteradas o crónicas del tendón distal del biceps brachii son infrecuentes. Su tratamiento es complejo por el grado de retracción y mala calidad del tendón lesionado, que no permiten una sutura directa. Describimos la reconstrucción con aloinjerto de tendón de Aquiles liofilizado mediante una nueva técnica y presentamos nuestra experiencia y resultados.

Material y método: Cuatro pacientes con actividades de alta demanda funcional, presentaban una rotura crónica (más de 3 meses de evolución) del tendón distal del biceps brachii. Se reconstruyó el tendón del biceps brachii con aloinjerto de Aquiles liofilizado mediante dos incisiones y reinserción en la tuberosidad bicipital con anclajes biodegradables.

Se valoran el arco de movilidad activo, resultado de la Mayo Elbow Performance Score (MEPS), dolor, fuerza y reincorporación laboral.

Resultados: A los 68 meses de seguimiento medio (rango: 36-88) todos los pacientes presentan un resultado satisfactorio, con un arco de movilidad activo completo, sin dolor y un resultado excelente de la MEPS. La fuerza en flexión y supinación fue similar
Objective: Inveterate or chronic distal biceps brachii tendon ruptures are rare. Treatment is complicated by shrinkage and poor quality of the injured tendon, which do not permit direct suture. We describe the reconstruction with lyophilized Achilles tendon allograft using a new technique and we present our experience and results.

Methods: Four patients with high functional demand activities, had a chronic tear (more than 3 months duration) of the distal biceps brachii tendon. An Achilles tendon allograft through a two-incision approach and biodegradable suture anchors were used to reconstruct the tendon.

Active range of motion, Mayo Elbow Performance Score (MEPS), pain, strength, and return to work were evaluated.

Results: At 68 months follow-up (range: 36-88) all patients have a satisfactory result with a full active range of motion, no pain and an excellent MEPS. The strength of flexion and supination was comparable with that of the contralateral side, although we observed thinning of the graft in the case with longer follow. All patients returned to the same job without restrictions at 14 weeks. One patient had posterior 
con respecto al lado contralateral, si bien se objetivó un ascenso de la masa muscular y adelgazamiento del injerto en el caso con mayor seguimiento. Todos los pacientes se reincorporaron a su mismo puesto de trabajo sin restricciones a las 14 semanas. Un paciente presentó una neuroapraxia del nervio interóseo posterior que se recuperó de forma satisfactoria a los 3 meses. No existieron otras complicaciones ni se precisaron reintervenciones.

Conclusiones: La reconstrucción con aloinjerto de tendón de Aquiles liofilizado en las roturas crónicas del tendón distal del biceps brachii presenta resultados satisfactorios, siendo una alternativa válida en pacientes con alta demanda funcional.

Palabras clave: tendón distal del biceps brachii, rotura crónica, injerto avascular, tendón Aquiles liofilizado. interosseous nerve neurapraxia that successfully recovered with conservative treatment at 3 months. No other complications or reoperations were required. Conclusions: Reconstruction of chronic disruptions of the distal biceps brachii tendon with a lyophilized Achilles tendon allograft shows satisfactory results and appears a valid alternative in patients with high functional demands.

Keywords: biceps brachii distal tendon, chronic disruption, avascular graft, lyophilized Achilles tendon.

\section{INTRODUCCIÓN}

L a rotura del tendón distal del biceps brachii (BB) es una lesión relativamente infrecuente. A pesar de su baja frecuencia, su incidencia se ha incrementado hasta en un $10 \%$ durante la última década, relacionándose la bursitis bicipital, el uso de esteroides y la nicotina como factores coadyuvantes para la aparición de esta rara lesión ${ }^{1}$.

En las roturas crónicas o inveteradas (más de 3 meses de evolución) distales del BB, la retracción y degeneración del tendón hace imposible su reparación anatómica.

Este tipo de lesiones presentan más dificultades para su tratamiento, principalmente por el proceso degenerativo distal y el grado de retracción tendinosa asociados que hacen dificultoso su reanclaje distal.

Se han descrito diversos procedimientos para el tratamiento, como la tenodesis al brachialis, injertos libres autólogos (semitendinosus, fascia lata y flexor carpi radialis) y aloinjertos.

Presentamos nuestra experiencia y resultados en la reconstrucción de las lesiones crónicas distales del tendón del BB, utilizando aloinjertos de tendón de Aquiles liofilizado.

\section{MATERIAL Y MÉTODO}

Entre los años 2004 y 2006 fueron intervenidos 4 pacientes con roturas crónicas ó inveteradas del tendón distal del BB mediante reconstrucción con aloinjerto (homoinjerto) de Aquiles liofilizado. En todos los casos el tiempo de evolución era mayor de 3 meses y se confirmó el diagnóstico mediante resonancia magnética. En un caso la lesión se localizaba a nivel de la unión musculo-tendinosa y el resto en el tendón distal.

La edad media de los cuatro pacientes era 38 años (rango: 24-52) y todos eran varones con trabajos de media-alta demanda funcional (Tabla I). El seguimiento medio fue 5,7 años (rango: 3-7,3). El miembro dominante estaba afectado en 2 de los 4 casos. Todos eran fumadores activos y ningún paciente presentaba historia de tratamiento con corticoesteroides o antibioterapia prolongada.

El arco de movilidad activa era completo y presentaban una asimetría evidente del aspecto de la cara anterior del brazo. Todos los casos tenían pérdida de fuerza para la flexión y supinación forzadas (inferior a 3/5) respecto al brazo contralateral. La medida de la fuerza se realizó 
TABla I - Datos de los Pacientes

\begin{tabular}{|c|c|c|c|c|c|c|c|}
\hline Caso & Sexo & $\begin{array}{c}\text { Edad } \\
\text { (años) }\end{array}$ & Lado & Dominancia & Puesto trabajo & Fuerza* & $\begin{array}{c}\text { Tiempo de } \\
\text { evolución } \\
\text { (meses) }\end{array}$ \\
\hline 1 & Varón & 24 & Derecho & Sí & Construcción & $2 / 5$ & 5 \\
\hline 2 & Varón & 34 & Izquierdo & No & Construcción & $3 / 5$ & 6 \\
\hline 3 & Varón & 52 & Izquierdo & No & Cadena de montaje & $2 / 5$ & 4 \\
\hline 4 & Varón & 42 & Izquierdo & Sí & Conductor & $2 / 5$ & 7 \\
\hline
\end{tabular}

${ }^{*}$ ) Fuerza en supinación y flexión comparada con el lado sano

con el paciente sentado con el codo flexionado $90^{\circ}$ y el antebrazo en rotación neutra.

\section{Técnica quirúrgica}

La reconstrucción se realiza mediante el abordaje de doble incisión modificado de la Clínica $\mathrm{Mayo}^{2}$.
Todos los procedimientos utilizados en el tratamiento fueron realizados tras la obtención de un consentimiento informado por parte del paciente, tanto de la cirugía como del aloinjerto.

Se coloca el paciente en decúbito supino con el manguito de isquemia lo más cerca de la axila posible, para poder movilizar con facilidad el
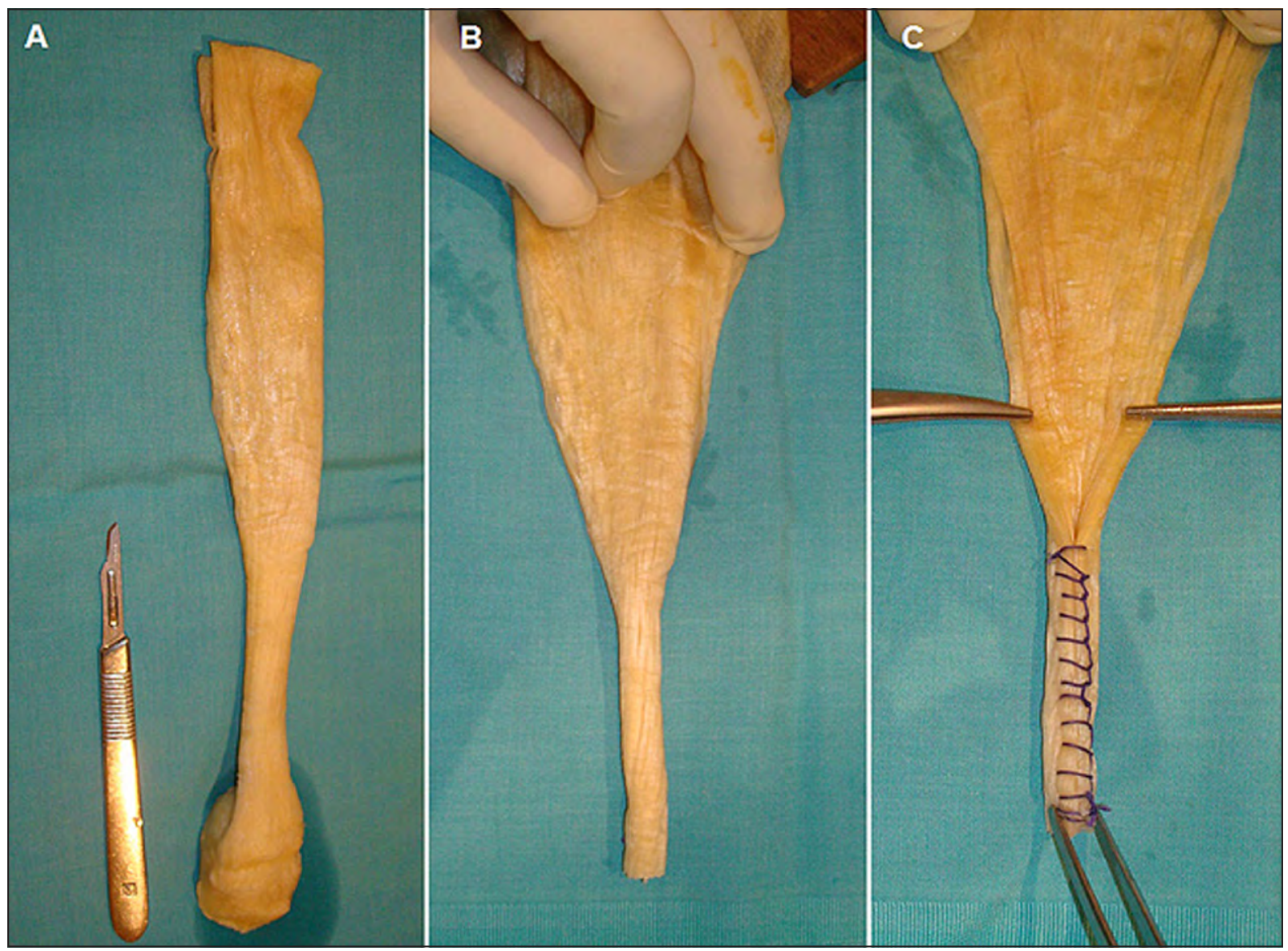

Figura 1. Tendón de Aquiles liofilizado con taco óseo en su inserción distal (A). Se reseca la porción ósea y cierre de los bordes en forma de cilindro con sutura contínua tipo Krackow. Visión dorsal (B) y palmar (C). 


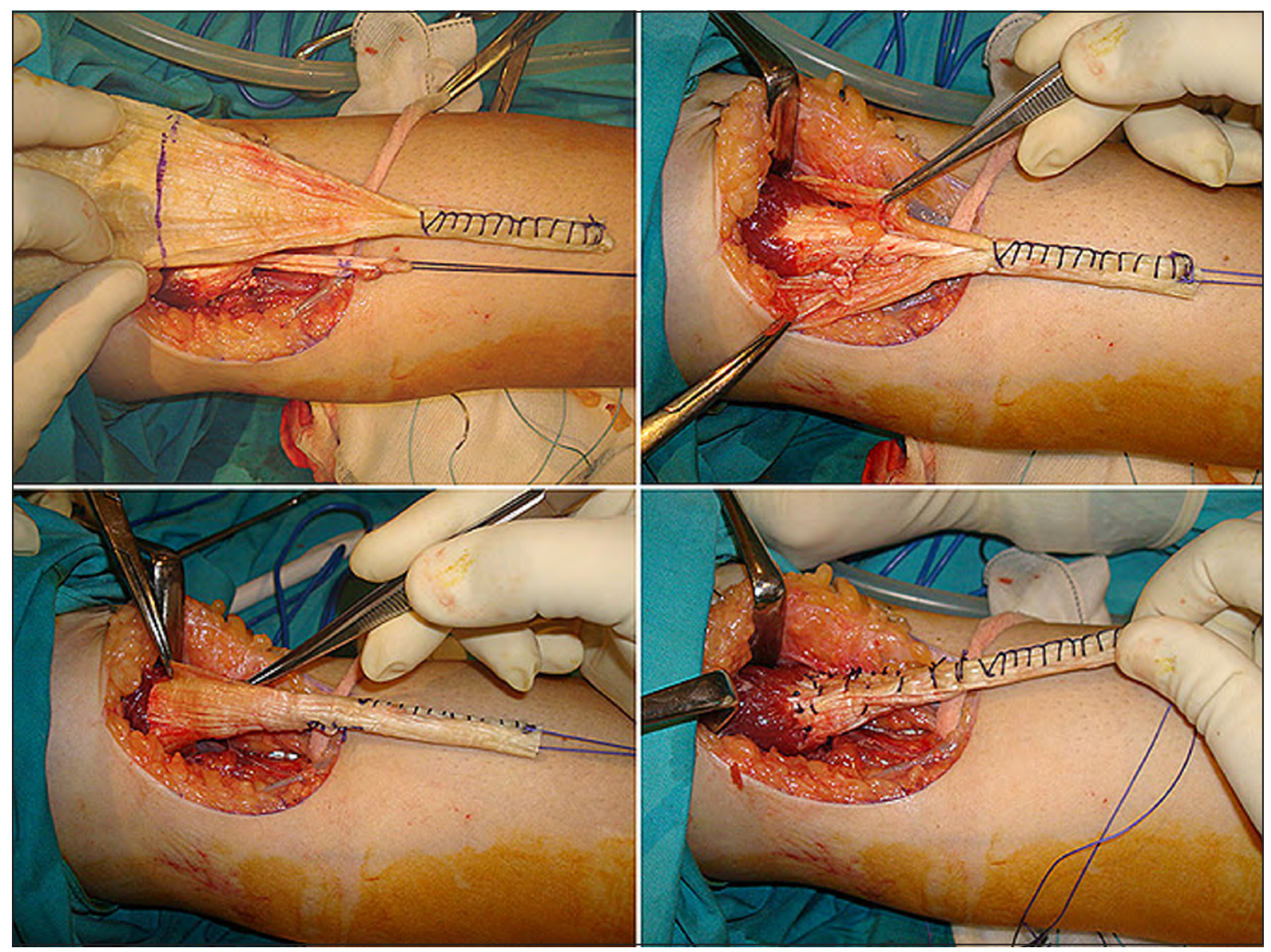

Figura 2. Colocación del aloinjerto sobre los restos del tendón del biceps brachii y medición del tamaño necesario. Sutura del injerto al tendón con puntos transfixiantes y aspecto del injerto al finalizar la sutura.

vientre muscular del BB. Previo a la intervención es necesario sumerger el tendón de Aquiles liofilizado en suero fisiológico al menos 2-3 horas antes de la cirugía, para que se hidrate y sea más sencillo su manipulación.

Bajo anestesia de plexo se realiza una incisión transversal a nivel del pliegue de flexión de la fosa antecubital del codo en forma de $\mathrm{S}$ itálica de 3-4 cm aproximadamente, con cuidado de no lesionar el nervio cutáneo antebraquial lateral. Se diseca de forma roma el intervalo muscular de distal a proximal hasta localizar el muñón distal del BB, que se libera y deja fiado en su extremo distal con una pinza de Allis, o con un par de puntos de tracción. Igualmente, hacia distal se intenta tunelizar de forma roma el canal del tendón bicipital hasta tocar la tuberosidad radial con el dedo con el antebrazo en máxima supinación. Se pasa una pinza recta pegada a la superficie del radio por su cara medial atravesando el intervalo muscular posterolateral con el antebrazo en máxima pronación y, protuyendo sobre la piel, se hace una incisión de unos 2-3 cm por la que se identificará la tuberosidad bicipital, que se deja libre de adherencias e inserciones. El cúbito no se expone para evitar la aparición de calcificaciones heterotópicas.

En una mesa aparte se extrae del suero fisiológico el aloinjerto, se secciona su parte más distal y el taco óseo que suele acompañarle. Elaboramos un cilindro distal de unos 5-6 cm con sutura reabsorbible $\mathrm{n}^{\mathrm{o}} 0$ y puntos de Krackow (Figura 1). La parte proximal abierta en abanico se coloca sobre el muñón tendinoso original y se suturan ambos con una sutura no reabsorbible de alta resistencia (nosotros utilizamos FiberWire ${ }^{\circledR} \mathrm{n}^{\circ} 2$ ) incluyendo la porción más distal de la unión músculotendinosa (Figura 2). Esta 


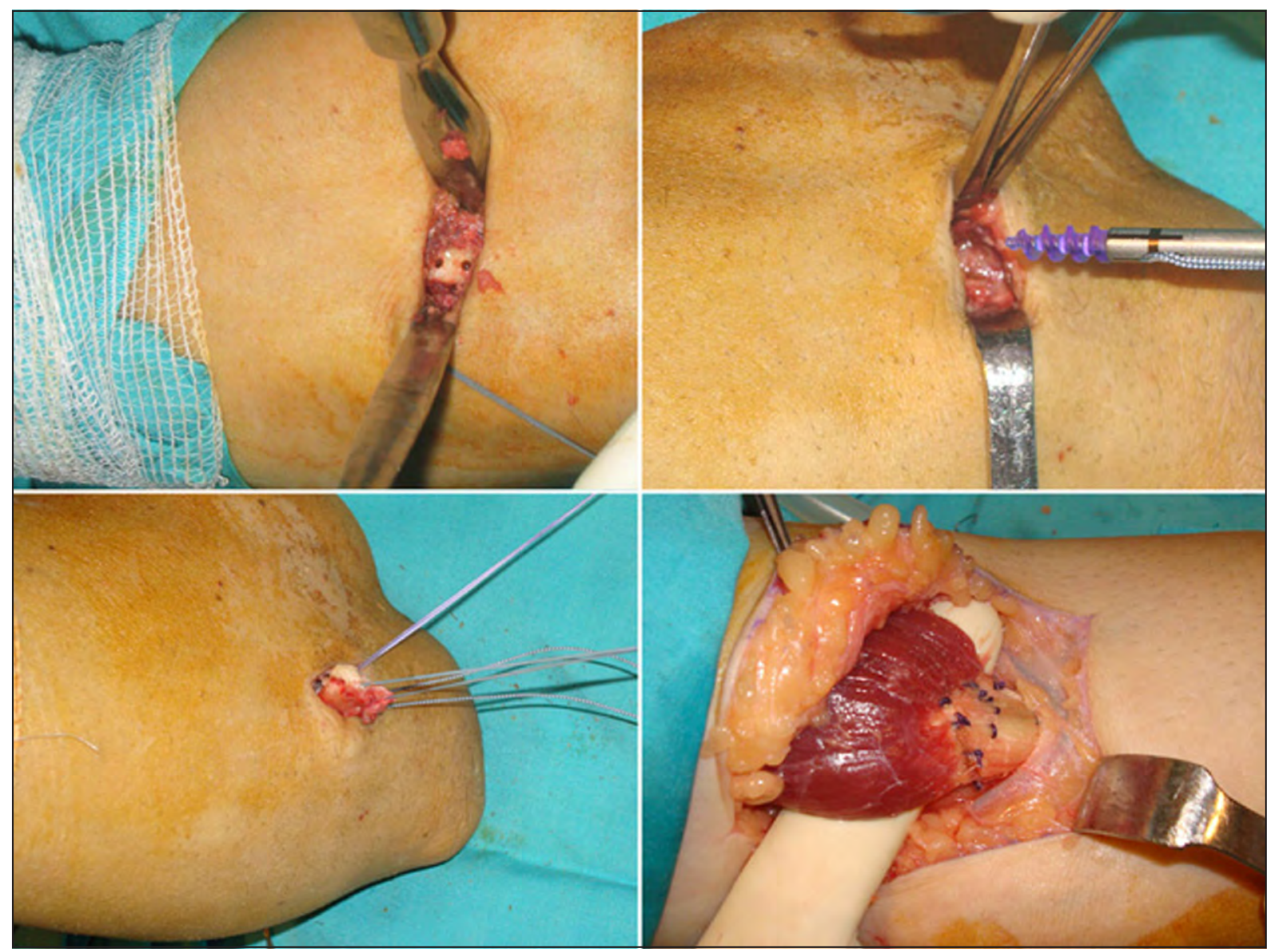

Figura 3. A través de la segunda vía se colocan los anclajes biodegradables en la tuberosidad bicipital del radio. Aspecto final y comprobación de la tensión del tendón tras la inserción distal del injerto.

sutura se refuerza con sutura reabsorbible $n^{\circ} 0$ hasta que sea necesario y se consiga una unión fuerte.

Una vez elongado el tendón, se coloca el brazo en flexión y pronación forzadas y se pasa por el túnel hasta la tuberosidad gracias a una pinza que se introduce desde la segunda incisión a la primera como si se tratara de una sutura primaria en un rotura aguda tratada por una técnica de doble vía convencional.

Una vez pasado el tendón colocamos dos arpones biodegradables (preferiblemente tipo atornillado) con el que fijamos la sutura en la parte más distal (Figura 3). Una vez realizada la reconstrucción dejamos un drenaje aspirativo tipo Redon y se inmoviliza en miembro con una férula posterior braquiopalmar a $90^{\circ}$ de flexión y pronosupinación neutra.
Todos los pacientes recibieron profilaxis antibiótica en el periodo postoperatorio inmediato mediante cefazolina 1 gramo cada 8 horas IV por 48 horas o clindamicina $600 \mathrm{mg}$ cada 12 horas IV por 48 horas en caso de alergia a penicilina o derivados, según protocolo de la comisión de infecciones del centro. No se realizó tratamiento alguno preventivo de las calcificaciones heterotópicas.

\section{Postoperatorio}

El brazo es inmovilizado por 4 semanas, tras las cuales se deja al paciente sin inmovilización alguna, y pasa a rehabilitación mediante medidas antiedema y antiinflamatorias, movilización pasiva y activa progresivas, potenciación muscular y maniobras de propiocepción por una duración mínima de 3 meses o hasta el alta laboral. 


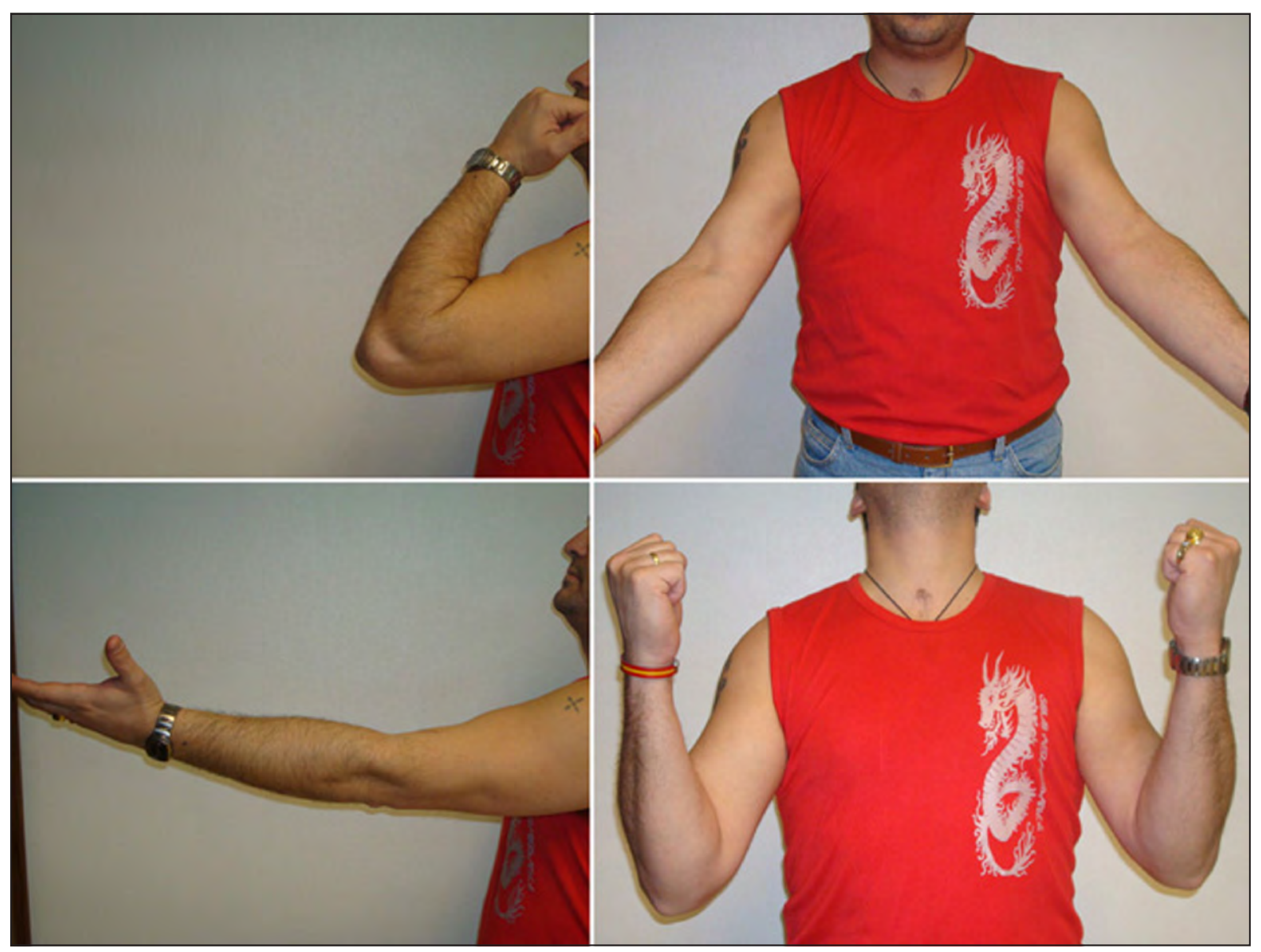

Figura 4. Exploración clínica del caso $n^{\circ} 4$ a los 36 meses del aloinjerto.

\section{Evaluación}

En todos los pacientes se valoraron la aparición de complicaciones, reintervenciones, el arco de movilidad activo, resultado de la Mayo Elbow Performance Score (MEPS), dolor, fuerza y reincorporación laboral.

Para valorar el dolor se utilizó la escala visual analógica (EVA). La fuerza en flexión y supinación fue evaluada de forma manual con el codo en $90^{\circ}$ de flexión y rotación neutra del antebrazo y comparada con el codo contralateral por un médico rehabilitador y utilizando la escala de Daniels que puntúa de 0 (sin función muscular) a 5 puntos (máxima función).

El resultado funcional fue valorado mediante la Mayo Elbow Performance Score ${ }^{3}$ que adjudica un máximo de 45 puntos para el dolor, 20 para la movilidad, 10 para la estabilidad y 25 para cinco actividades de la vida diaria (Tabla II).
Se considera un resultado excelente cuando se obtienen puntuaciones $>89$ puntos, bueno entre 75-89 puntos, regular entre 60-74 puntos y malo para puntuaciones $<60$ puntos.

El resultado laboral se analizó valorando los días de baja totales y la medición de las secuelas e incorporación a su trabajo, siguiendo la escala de valoración del Instituto Nacional de la Salud (INS) que determina las categorías de curado, secuelas con baremo, incapacidad permanente parcial (IPP), incapacidad permanente total (IPT), incapacidad absoluta (IA) y gran invali$\operatorname{dez}(\mathrm{GI})^{4}$.

\section{RESULTADOS}

No se apreciaron complicaciones menores ni mayores, excepto un paciente que presentó una neuroapraxia del nervio interóseo posterior, 


\section{TABLA II - Resultado POstoperatorio}

\begin{tabular}{|c|c|c|c|c|c|c|c|c|c|}
\hline Caso & Complicaciones & $\begin{array}{c}\text { EVA (1) } \\
(\mathbf{0}-\mathbf{1 0})\end{array}$ & $\begin{array}{c}\text { Arco } \\
\text { FE}^{2}\left({ }^{(}\right)\end{array}$ & $\begin{array}{c}\text { Arco } \\
\text { rotación (0) }\end{array}$ & MEPS & $\begin{array}{c}\text { Fuerza } \\
\text { (0-5) }\end{array}$ & $\begin{array}{c}\text { Baja laboral } \\
\text { (semanas) }\end{array}$ & $\begin{array}{c}\text { Mismo } \\
\text { puesto de } \\
\text { trabajo }\end{array}$ & $\begin{array}{c}\text { Seguimiento } \\
\text { (meses) }\end{array}$ \\
\hline 1 & No & 0 & 140 & 170 & 100 & $4 / 5$ & 14 & Sí & 88 \\
\hline 2 & No & 0 & 140 & 170 & 100 & $5 / 5$ & 10 & Sí & 78 \\
\hline 3 & $\begin{array}{c}\text { Neurapraxia } \\
\text { transitoria de } \\
\text { NIP (3) }\end{array}$ & 0 & 135 & 165 & 90 & $4 / 5$ & 18 & Sí & 69 \\
\hline 4 & No & 0 & 140 & 170 & 90 & $5 / 5$ & - & Sí & 36 \\
\hline
\end{tabular}

(1) EVA: Escala Visual Analógica del dolor. (2) Arco de FE: Arco de flexo-extensión

(3) NIP: Nervio interóseo posterior. (4) MEPS: Mayo Elbow Performance Score

\section{TABLA III - Resultados comparados}

\begin{tabular}{|c|c|c|c|c|c|c|c|c|}
\hline Serie & $\begin{array}{c}\mathbf{N}^{\circ} \\
\text { pacientes }\end{array}$ & $\begin{array}{l}\text { Seguimiento } \\
\text { (meses) }\end{array}$ & FLX & EXT & SUP & PRN & Fuerza & MEPS \\
\hline Sanchez-Sotelo et al, $2002^{13}$ & 4 & 29 & 136 & 0 & 84 & 83 & $5 / 5$ & 100 \\
\hline Darlis y Sotereanos, $2006^{14}$ & 7 & 29 & 145 & 20 & 83 & 86 & $5 / 5$ & 97 \\
\hline Patterson et al, $2009^{21}$ & 1 & 5.5 & 135 & 0 & 75 & 70 & $5 / 5$ & N/D \\
\hline Serie presentada, 2012 & 4 & 68 & 139 & 0 & 83 & 87 & $4.5 / 5$ & 95 \\
\hline
\end{tabular}

Arcos de movilidad expresados en grados.

FLX: flexión; EXT: extensión; SUP: supinación; PRN: pronación.

N/D: no descrito.

MEPS: Mayo Elbow Performance Score.

que se resolvió con tratamiento sintomático sin secuelas a los 3 meses. Ningún paciente precisó nuevas intervenciones (Tabla III).

Todos los pacientes estaban satisfechos con el procedimiento y volvieron a su vida activa, laboral y deportiva sin restricciones. El arco de movilidad fue completo respecto al lado contralateral (Figura 4) para la flexo-extensión y la prono-supinación. No presentaban dolor y se obtuvo una media de 95 puntos en la MEPS.

La fuerza en flexión y supinación se incrementó respecto al preoperatorio. Fue comparable con el lado contralateral sano (5/5) en dos casos y levemente menor en los otros dos (4/5). En el paciente con mayor seguimiento (8 años) se objetivó un ascenso progresivo de la masa muscular bicipital con adelgazamiento del injerto (Figura 5). No presentaba roturas del mismo y la función era aceptable, pero con una objetivable pérdida de fuerza para la flexión y supinación combinadas.

En la evaluación laboral, el periodo de baja laboral medio tras la cirugía fue de 14 semanas (rango: 10-18). Todos los pacientes volvieron a 


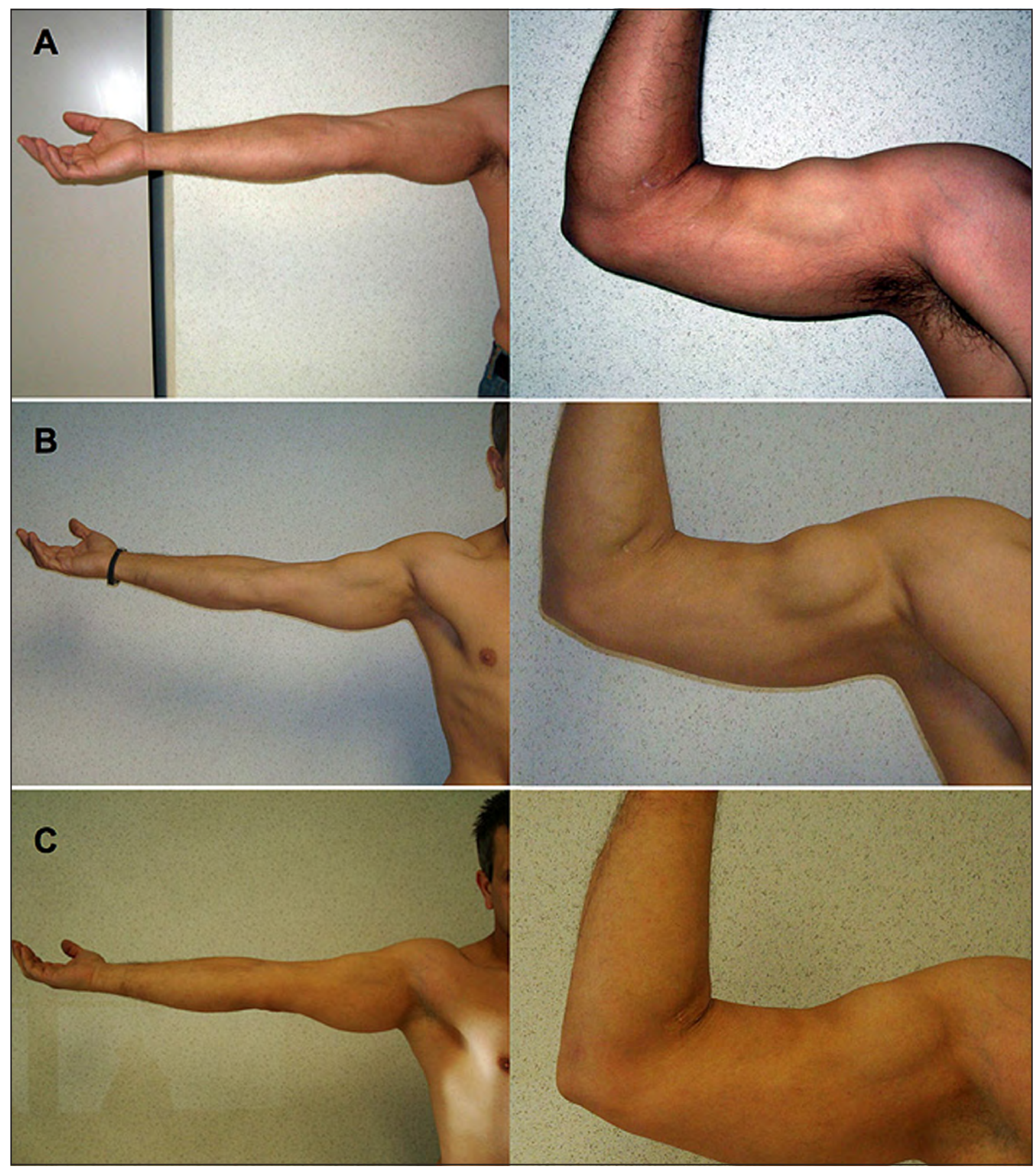

Figura 5. Exploración clínica del caso $n^{o} 1$ al año (A), a los 4 años (B) y a los 7 años (C) del aloinjerto, donde se aprecia un ascenso progresivo de la masa muscular del biceps brachii.

trabajar a su mismo puesto de trabajo sin ningún tipo de restricciones.

Siguiendo la escala de valoración laboral ningún paciente presentó secuelas que justificaron la obtención de una incapacidad permanente parcial o total. No se concedieron incapacidades absolutas ni grandes incapacidades.

\section{DISCUSIÓN}

Los pacientes con lesiones inveteradas del tendón distal del BB presentan una asimetría del contorno del brazo y disminución de la función global del codo. En numerosas ocasiones presentan dolor de tipo neurítico por afectación 
del nervio cutáneobraquial lateral o del musculocutáneo, siendo esta una de las causas más frecuentes de intervención quirúrgica.

En pacientes sintomáticos la reparación no anatómica mediante la tenodesis distal del BB al brachialis no restaura la fuerza de supinación del codo. Klonz et $\mathrm{al}^{5}$ analizaron de forma retrospectiva mediante estudios isocinéticos 14 pacientes tratados quirúrgicamente por roturas distales del $\mathrm{BB}$ mediante reinserción anatómica $(n=6)$ y reparación no anatómica mediante tenodesis al brachialis $(n=8)$. A los 65 meses de seguimiento medio observaron una pérdida de fuerza en supinación del 42-56\% en la mitad de los casos tratados con tenodesis del $\mathrm{BB}$ al brachialis respecto a los que se realizó una reparación anatómica. Esta pérdida de fuerza convierte a esta técnica en un procedimiento subóptimo e inaceptable en deportistas o pacientes de alta demanda funcional.

La reconstrucción diferida mediante ligamentoplastias es una alternativa a este tipo de reparaciones. Han sido descritos la utilización de fascia lata ${ }^{6}$, semitendinosus ${ }^{7}$, palmaris longus ${ }^{8}$, flexor carpi radialis ${ }^{9}$ e injertos sintéticos aisla$\operatorname{dos}^{10}$ o con fascia lata ${ }^{11}$, cuyos resultados han sido publicados en series pequeñas o como casos clínico. La reconstrucción con tendón del semitendinosus es la técnica que tiene más trabajos publicados y es la más utilizada para reconstruir este tipo de lesiones con un buen resultado funcional $^{7}$. Sin embargo, este procedimiento asocia una morbilidad adicional de la zona donante y, en ocasiones, la longitud del injerto del tendón puede ser insuficiente, especialmente si el grado de retracción del BB es grande o si la rotura ocurrió a nivel de la unión músculo tendinosa.

La alternativa a estos procedimientos son los injertos libres. El aloinjerto de Aquiles presenta una serie de atractivas ventajas que permiten salvar estas incidencias con respecto al semitendinoso. Presenta menor morbilidad, aporta más tejido para la reconstrucción y presenta unas excelentes propiedades biomecánicas que han sido demostrados mediante estudios «in vivo» en reconstrucciones de rodilla y tobillo ${ }^{12}$.

Sánchez Sotelo et $\mathrm{al}^{13}$ publicaron los resultados de 4 pacientes con roturas crónicas distales del BB tratados en la Clínica Mayo mediante aloinjerto de Aquiles con taco óseo por doble vía de abordaje y fijación mediante puntos transóseos en la tuberosidad bicipital. Unos años más tarde, Darlis y Sotereanos ${ }^{14}$ presentaron la misma técnica en 7 pacientes, pero sin taco óseo, única vía de abordaje y arpones metálicos en la tuberosidad bicipital.

Boyd y Anderson ${ }^{15}$ describieron la reinserción por doble vía que evitaba los riesgos inherentes a la disección de la fosa antecubital. Sin embargo, la disección de la musculatura de la cara externa del olécranon incrementa las calcificaciones heterotópicas, hecho que se vio disminuido tras la modificación de la vía de abordaje realizada por Morrey ${ }^{2}$.

Si bien no existen diferencias a largo plazo entre realizar la sutura por una vía o mediante doble abordaje ${ }^{16}$, la doble vía necesita una menor incisión y exposición, con lo que se disminuye la morbilidad del procedimiento y reproduce mejor el recorrido natural del tendón en torno a la tuberosidad radial durante el arco de rotación del antebrazo.

Con respecto al sistema de fijación, la utilización de arpones ha demostrado una buena resistencia contrastada en otras localizaciones, así como en suturas primarias del tendón distal del $\mathrm{BB}^{17}$.

Existen numerosos estudios biomecánicos recientes en cadáver que valoran la fuerza de fijación en el hueso. La sutura transósea presenta la mayor fuerza de fijación cuando se compara con la fijación con un arpón simple ${ }^{18}$. Sin embargo, cuando se compara este sistema con la fijación mediante dos arpones dobles, la resistencia es superior con respecto a la fijación transósea ${ }^{19,20}$.

Aparte de la fuerza de fijación y resistencia contrastadas, este tipo de sistemas facilita la reinserción cuando esta se realiza por doble vía.

En nuestros casos hemos hecho una modificación técnica, realizando la reconstrucción con injerto de Aquiles liofilizado sin taco óseo, mediante doble abordaje y fijación distal en la tuberosidad con arpones biodegradables. La variante técnica que hemos utilizado aprovecha las ventajas de la doble vía de abordaje (menor riesgo de lesionar estructuras importantes con menor disección de la fosa antecubital) y simplifica la técnica de fijación al utilizar arpones óseos. Con la utilización de aloinjertos se reduce la morbilidad secundaria asociada a los injertos tendinosos 
autólogos, por lo que es nuestra técnica de elección. No obstante, otros sistemas utilizados en las lesiones agudas pueden ser utilizadas en este tipo de lesiones. Patterson et $\mathrm{al}^{21}$ publicaron un paciente con rotura crónica distal del BB tratado con aloinjerto de Aquiles, donde se utilizó el sistema EndoButton para su fijación con buenos resultados clínicos a los 5 meses y medio.

Nuestra indicación principal para realizar la reconstrucción de roturas crónicas del tendón distal del BB son pacientes jóvenes, de alta demanda funcional, en edad activa laboral o deportiva, en los que es imposible aproximar el muñón distal del biceps a la tuberosidad bicipital con el codo en extensión de unos 40-50 .

Si bien nuestros resultados preliminares son buenos y similares a las principales series publicadas (Tabla IV), no creemos que sea una técnica de uso generalizado.

Los trabajos publicados son muy esca$\operatorname{sos}^{13,14,21}$. Se tratan de estudios de pocos pacientes y con un seguimiento a corto o medio plazo. No sabemos qué ocurre con estos injertos a largo plazo. Todos los aloinjertos eran liofilizados. La liofilización es un proceso en el que se congela el producto y posteriormente se introduce en una cámara de vacío para realizar la separación del agua por sublimación. De esta manera se elimina el agua desde el estado sólido al gaseoso del ambiente sin pasar por el estado líquido. Para acele- rar el proceso se utilizan ciclos de congelaciónsublimación con los que se consigue eliminar prácticamente la totalidad del agua libre contenida en el producto original, pero preservando la estructura molecular de la sustancia liofilizada. Este método de congelación-secado altera de forma drástica las propiedades mecánicas del tejido de un aloinjerto más que las técnicas de congelación en fresco. En nuestra experiencia hemos observado una retracción del vientre muscular y un adelgazamiento del tendón de forma progresiva en los casos con mayor tiempo desde la plastia (Figura 5). Este dato no ha sido descrito en las series publicadas que presentan un menor seguimiento a largo plazo y podría tener relación con el método descrito de liofilizado.

Es importante realizar una correcta valoración individual, teniendo en cuenta la edad, dominancia, la actividad laboral y/o deportiva y evaluar las articulaciones vecinas proximales y distales. Por otro lado, hay que tener presente la potencial degradación que ocurre con el tiempo con el aloinjerto liofilizado.

En resumen, la reconstrucción con aloinjerto de Aquiles en las roturas crónicas distales del BB presenta unos resultados clínicos y laborales satisfactorios y la técnica presentada (doble vía + arpones) es una alternativa válida en pacientes con alta demanda funcional y roturas crónicas del tendón del BB.

\section{BIBLIOGRAFÍA}

1. Safran MR, Graham SM. Distal biceps tendon ruptures: incidence, demographics and the effect of smoking. Clin Orthop, 2002; 404: 275-83.

2. Morrey BF, Askew LJ, An KN, Dobyns JH. Rupture of the distal biceps tendon: biomechanical assessment of different treatment options. J Bone Joint Surg Am, 1985; 67: 418-26.

3. Morrey BF, An K. Functional evaluation of the elbow. En: Morrey $B F(E d)$. The Elbow and its Disorders. 3rd Ed. Philadelphia: WB Saunders; 2000: 74-83.

4. Hevia-Campomanes E, Miranda F, Vivanco MC, Gómez F. Los accidentes de trabajo y las enfermedades profesionales. Gestión, prestaciones, procedimiento y jurisprudencia. Madrid: Colex, 1990.

5. Klonz A, Loitz D, Wöhler P, Reilmann H. Rupture of the distal biceps brachii tendon: isokinetic power analisis and complications after anatomic reinsertion compared with fixation to the brachialis muscle. J Shoulder Elbow Surg, 2003; 12: 607-11.

6. Hovelius L, Josefsson G. Rupture of the distal biceps tendon: report of five cases. Acta Orthop Scand, 1977; 48: 280-2.

7. Hang DW, Bach BR, Bajchuk J. Repair of chronic distal biceps brachii tendon rupture using free autogenous semitendinosus tendon. Clin Orthop, 1996; 323: 188-91.

8. Rantanen J, Orava S. Rupture of the distal biceps tendon. A report of 19 patients treated with anatomic reinsertion, and a meta-analysis of 147 cases found in the literature. Am J Sports Med, 1999; 27: 128-32.

9. Levy HJ, Mashoof AA, Morgan D. Repair of chronic ruptures of the distal biceps tendon using flexor carpi radialis tendon graft. Am J Sports Med, 2000; 28: 538-40.

10. Morrey BF. Injury of the flexors of the elbow: biceps tendon 
injury. En: Morrey BF (Ed). The Elbow and its Disorders. 3rd ed. Philadelphia: WB Saunders; 2000: 468-78.

11. Kaplan FT, Rokito AS, Birdzell MG, Zuckerman JD. Reconstruction of chronic distal biceps tendon rupture with the use of fascia lata combined with a ligament augmentation device: a report of 3 cases. J Shoulder Elbow Surg, 2002; 11: 633-6.

12. Levitt RL, Malinin T, Posada A, Michalow A. Reconstruction of anterior cruciate ligaments with bone-patellar tendon-bone and Achilles tendon allografts. Clin Orthop, 1994; 303: 67-78.

13. Sánchez-Sotelo J, Morrey BF, Adams RA, O'Driscoll SW. Reconstruction of chronic ruptures of the distal biceps tendon with use of an Achilles tendon allograft. J Bone Joint Surg Am, 2002; 84: 999-1005.
14. Darlis NA, Sotereanos DG. Distal biceps tendon reconstruction in chronic ruptures. J Shoulder Elbow Surg, 2006; 15: 614-9.

15. Boyd HB, Anderson LD. A method for reinsertion of the distal biceps brachii tendon. J Bone Joint Surg Am, 1961; 43: 1041-3.

16. El-Hawary R, MacDermid J, Faber K, Patterson S, King G. Distal biceps tendon repair: comparison of surgical techniques. J Hand Surg Am, 2003; 28: 496-502.

17. Ozyrekaglu T, Tsai TM. Ruptures of the distal biceps brachii tendon: results of three surgical techniques. Hand Surg, 2003; 8: 65-73.

18. Ketter M, Tingart MJ, Lunger J, Kuhn V. Reattachment of the distal tendon of biceps. Factors affecting the failure strength of the repair. J Bone Joint Surg Br, 2008; 90: 103-6.

19. Pereira D, Kvitne R, Liang M, Giacobetti F, Ebramzedeh E. Surgical repair of distal biceps tendon ruptures: a biomechanical comparison of two techniques. Am J Sports Med, 2002; 30: 432-6.

20. Lemos SE, Ebramzedeh E, Kvitne RS. A new technique: in vitro suture anchor fixation has superior yield strength to bone tunnel fixation for distal biceps tendon repair. Am J Sports Med, 2004; 32: 406-10.

21. Patterson RW, Sharma J, Lawton JN, Evans PJ. Distal biceps tendon reconstruction with tendoachilles allograft: a modification of the Endobutton technique utilizing an ACL reconstruction system. $\mathrm{J}$ Hand Surg Am, 2009; 34: 545-52. 